\title{
Gas-to-liquid Phase Transition of PAH at Flame Temperatures
}

Peng Liu, Yang Li*, S. Mani Sarathy, William L. Roberts

King Abdullah University of Science and Technology (KAUST), Clean Combustion Research Center, Thuwal 23955-

6900, Saudi Arabia

\section{Statistical comparison}

Table S1. Key parameters of the Bland-Altman plot.

Key parameters

Definition

Number of data points

$n\left(\operatorname{Exp}_{1}, \operatorname{Exp}_{2}, \ldots, \operatorname{Exp}_{n}\right.$ and $\left.\mathrm{Cal}_{1}, \mathrm{Cal}_{2}, \ldots, \mathrm{Cal}_{n}\right)$

Degree of freedom

$v=n-1$

Student's t-value

$t=f(v$, Probability level $)$

Average values

$X_{i}=\left(\operatorname{Exp}_{i}+\operatorname{Cal}_{i}\right) / 2$

Differences

$d_{i}=\left(\operatorname{Exp}_{i}-\operatorname{Cal}_{i}\right)$

The mean difference or bias

$\bar{d}=\sum_{i}^{n}\left(\operatorname{Exp}_{i}-\operatorname{Cal}_{i}\right) / n=\sum_{i}^{n}\left(d_{i}\right) / n$

Sample standard deviation

$s_{d}=\sqrt{\frac{\sum\left(d_{i}-\bar{d}\right)}{(n-1)}}$

95\% Limits of agreement

$\bar{d} \pm 1.96 s_{d}$

Error bars for bias

$t \times s_{d} / \sqrt{n}$

Error bars for limits of agreement

$1.709 \times t \times s_{d} / \sqrt{n}$ 
Table S2. The key parameters of the Bland-Altman plot shown in Fig. 1.

\begin{tabular}{cccc}
\hline Key parameters & Calculation 1 & Calculation 2 & Calculation 3 \\
\hline Number of data points & 20 & \\
Degree of freedom & 19 & \\
Student's t-value & 2.40 & 1.73 & 0.22 \\
The mean difference or bias & 2.44 & 1.46 & 1.72 \\
Sample standard deviation & 0.94 & 2.05 & 0.67 \\
Error bars for bias & 1.61 & 0.79 & 1.14 \\
\hline
\end{tabular}

Table S3. Comparisons of constant pressure heat capacities $\left(\mathrm{C}_{\mathrm{p}}(298 \mathrm{~K})\right.$, cal $\left./ \mathrm{mol} / \mathrm{K}\right)$ predicted by DFT/B3LYP/6-311+(g,d), DFT/M026X/6-311++G(d,p) and the values from NIST chemistry webbook.

\begin{tabular}{|l|l|l|l|}
\hline Species & B3LYP/6-311+(g,d) & M026X/6-311++G(d,p) & NIST data \\
\hline 1-methylnaphthalene & 38.15888 & 37.61245 & 38.07361 \\
\hline 2-methylnaphthalene & 38.28488 & 36.69426 & 36.95029 \\
\hline Acenaphthene & 38.80688 & 37.86101 & 38.76673 \\
\hline Acenaphthylene & 35.52588 & 35.95493 & 36.99809 \\
\hline Anthracene & 44.41088 & 43.29353 & 44.14436 \\
\hline Benz[a]anthracene & 56.53788 & 55.15594 & 55.99904 \\
\hline Benzene & 19.91488 & 19.3818 & 19.70363 \\
\hline Biphenyl & 39.85788 & 36.98597 & 39.84226 \\
\hline Chrysene & 56.58188 & 55.10143 & 56.23805 \\
\hline Fluoranthene & 48.82288 & 47.60776 & 48.66157 \\
\hline Fluorene & 41.64388 & 40.64595 & 31.24059 \\
\hline Naphthalene & 32.07988 & & 59254 \\
\hline
\end{tabular}




\begin{tabular}{|c|c|c|c|}
\hline Phenanthrene & 44.27788 & 43.2031 & 44.38337 \\
\hline Pyrene & 48.64388 & 47.47444 & 48.80497 \\
\hline Naphthacene & 56.75288 & 55.36618 & 55.78394 \\
\hline Ethenylbenzene & 29.08188 & 28.11373 & 28.7261 \\
\hline Ethylbenzene & 31.01288 & 30.51036 & 30.44933 \\
\hline Indene & 29.87288 & 29.11836 & 29.43117 \\
\hline Isopropenylbenzene & 34.68888 & 33.77177 & 33.57792 \\
\hline Toluene & 26.13388 & 24.53556 & 24.78489 \\
\hline Methane & 8.544878 & 8.448878 & 8.530115 \\
\hline Ethane & 12.37688 & 12.04788 & 12.54541 \\
\hline Propane & 17.28788 & 16.72988 & 17.59082 \\
\hline Propene & 15.38588 & 14.92888 & 15.37285 \\
\hline 1-Butene & 20.32588 & 19.63788 & 20.44933 \\
\hline Cyclopentane & 21.43788 & - & 19.78967 \\
\hline Phenylethyne & 28.05788 & 26.87888 & 27.45937 \\
\hline Triphenylene & 56.53988 & - & 56.52486 \\
\hline Biphenylene & 37.48988 & 35.93488 & 38.07361 \\
\hline Coronene $^{\mathrm{a}}$ & 66.59288 & 66.40588 & 67.30402 \\
\hline Ovalene $^{\mathrm{a}}$ & 86.95588 & 86.92488 & 88.24092 \\
\hline
\end{tabular}

-convergence failure

a optimization using the DFT/B3LYP/6-311(g,d) and DFT/M026X/6-311G(d,p) methods. 


\section{Geometries of all calculated species:}

\begin{tabular}{lccc}
\multicolumn{4}{l}{ 1-methylnaphthalene } \\
C & 2.757378000 & -0.174865000 & 0.000000000 \\
$\mathrm{C}$ & 1.848140000 & -1.198820000 & 0.000000000 \\
$\mathrm{C}$ & 0.454024000 & -0.936623000 & 0.000000000 \\
$\mathrm{C}$ & 0.000000000 & 0.411417000 & 0.000000000 \\
$\mathrm{C}$ & 0.968925000 & 1.449798000 & 0.000000000 \\
$\mathrm{C}$ & 2.310115000 & 1.165624000 & 0.000000000 \\
$\mathrm{H}$ & -0.137391000 & -3.017074000 & 0.000000000 \\
$\mathrm{H}$ & 3.819747000 & -0.387699000 & 0.000000000 \\
$\mathrm{H}$ & 2.181069000 & -2.231341000 & 0.000000000 \\
$\mathrm{C}$ & -0.494492000 & -1.992982000 & 0.000000000 \\
$\mathrm{C}$ & -1.405435000 & 0.680455000 & 0.000000000 \\
$\mathrm{H}$ & 0.642063000 & 2.482490000 & 0.000000000 \\
$\mathrm{H}$ & 3.033933000 & 1.972048000 & 0.000000000 \\
$\mathrm{C}$ & -2.283111000 & -0.374777000 & 0.000000000 \\
$\mathrm{C}$ & -1.832642000 & -1.716329000 & 0.000000000 \\
$\mathrm{H}$ & -3.349700000 & -0.176898000 & 0.000000000 \\
$\mathrm{H}$ & -2.557961000 & -2.521387000 & 0.000000000 \\
$\mathrm{C}$ & -1.908576000 & 2.099430000 & 0.000000000 \\
$\mathrm{H}$ & -1.559691000 & 2.643764000 & 0.881660000 \\
$\mathrm{H}$ & -1.559691000 & 2.643764000 & -0.881660000 \\
$\mathrm{H}$ & -2.998332000 & 2.118369000 & 0.000000000
\end{tabular}

2-methylnaphthalene

$\begin{array}{lrrc}\mathrm{C} & -2.952039000 & 0.344681000 & 0.000007000 \\ \mathrm{C} & -1.913025000 & 1.237713000 & 0.000011000 \\ \mathrm{C} & -0.569288000 & 0.784885000 & 0.000005000 \\ \mathrm{C} & -0.309383000 & -0.610787000 & -0.000007000 \\ \mathrm{C} & -1.405372000 & -1.511567000 & -0.000012000 \\ \mathrm{C} & -2.694181000 & -1.046570000 & -0.000005000 \\ \mathrm{H} & 0.338319000 & 2.748165000 & 0.000013000 \\ \mathrm{H} & -3.975659000 & 0.699786000 & 0.000012000 \\ \mathrm{H} & -2.103739000 & 2.305712000 & 0.000019000 \\ \mathrm{C} & 0.532778000 & 1.680788000 & 0.000008000 \\ \mathrm{C} & 1.037271000 & -1.062944000 & -0.000014000 \\ \mathrm{H} & -1.204589000 & -2.577692000 & -0.000021000 \\ \mathrm{H} & -3.523444000 & -1.744047000 & -0.000008000 \\ \mathrm{C} & 2.090290000 & -0.185430000 & -0.000008000 \\ \mathrm{C} & 1.816057000 & 1.210424000 & 0.000002000 \\ \mathrm{H} & 1.223337000 & -2.132921000 & -0.000026000 \\ \mathrm{H} & 2.648675000 & 1.906707000 & 0.000002000 \\ \mathrm{C} & 3.519999000 & -0.656172000 & 0.000008000 \\ \mathrm{H} & 4.050248000 & -0.285290000 & -0.880902000 \\ \mathrm{H} & 4.050119000 & -0.285603000 & 0.881128000 \\ \mathrm{H} & 3.578086000 & -1.744944000 & -0.000178000\end{array}$




\section{acenaphthene}

$\begin{array}{lrrr}\mathrm{C} & 0.000000000 & 2.418034000 & 1.183426000 \\ \mathrm{C} & 0.000000000 & 1.272894000 & 1.945743000 \\ \mathrm{C} & 0.000000000 & 0.000000000 & 1.315567000 \\ \mathrm{C} & 0.000000000 & 0.000000000 & -0.088178000 \\ \mathrm{C} & 0.000000000 & 1.171949000 & -0.871124000 \\ \mathrm{C} & 0.000000000 & 2.387247000 & -0.238667000 \\ \mathrm{H} & 0.000000000 & -1.336827000 & 3.028320000 \\ \mathrm{H} & 0.000000000 & 3.381721000 & 1.680114000 \\ \mathrm{H} & 0.000000000 & 1.336827000 & 3.028320000 \\ \mathrm{C} & 0.000000000 & -1.272894000 & 1.945743000 \\ \mathrm{C} & 0.000000000 & -1.171949000 & -0.871124000 \\ \mathrm{H} & 0.000000000 & 3.318214000 & -0.794536000 \\ \mathrm{C} & 0.000000000 & -2.387247000 & -0.238667000 \\ \mathrm{C} & 0.000000000 & -2.418034000 & 1.183426000 \\ \mathrm{H} & 0.000000000 & -3.318214000 & -0.794536000 \\ \mathrm{H} & 0.000000000 & -3.381721000 & 1.680114000 \\ \mathrm{C} & 0.000000000 & -0.780911000 & -2.336179000 \\ \mathrm{C} & 0.000000000 & 0.780911000 & -2.336179000 \\ \mathrm{H} & -0.878330000 & 1.180538000 & -2.847625000 \\ \mathrm{H} & -0.878330000 & -1.180538000 & -2.847625000 \\ \mathrm{H} & 0.878330000 & 1.180538000 & -2.847625000 \\ \mathrm{H} & 0.878330000 & -1.180538000 & -2.847625000\end{array}$

\section{acenaphthylene}

$\begin{array}{lrrr}\mathrm{C} & 2.420290000 & -1.102537000 & 0.000002000 \\ \mathrm{C} & 1.279877000 & -1.874934000 & -0.000015000 \\ \mathrm{C} & 0.000000000 & -1.250793000 & -0.000023000 \\ \mathrm{C} & 0.000000000 & 0.137815000 & -0.000023000 \\ \mathrm{C} & 1.158481000 & 0.946324000 & -0.000002000 \\ \mathrm{C} & 2.381156000 & 0.321749000 & 0.000013000 \\ \mathrm{H} & -1.355282000 & -2.956915000 & -0.000022000 \\ \mathrm{H} & 3.387460000 & -1.591441000 & 0.000011000 \\ \mathrm{H} & 1.355282000 & -2.956915000 & -0.000019000 \\ \mathrm{C} & -1.279877000 & -1.874934000 & -0.000018000 \\ \mathrm{C} & -1.158481000 & 0.946324000 & -0.000005000 \\ \mathrm{H} & 3.310507000 & 0.880553000 & 0.000035000 \\ \mathrm{C} & -2.381156000 & 0.321749000 & 0.000007000 \\ \mathrm{C} & -2.420291000 & -1.102537000 & -0.000004000 \\ \mathrm{H} & -3.310507000 & 0.880553000 & 0.000027000 \\ \mathrm{H} & -3.387460000 & -1.591441000 & 0.000003000 \\ \mathrm{C} & -0.677539000 & 2.341183000 & 0.000022000 \\ \mathrm{C} & 0.677539000 & 2.341183000 & 0.000026000 \\ \mathrm{H} & 1.312643000 & 3.216022000 & 0.000042000 \\ \mathrm{H} & -1.312643000 & 3.216022000 & 0.000036000\end{array}$




\section{anthracene}

$\begin{array}{lrrr}\mathrm{C} & 3.645689000 & -0.713759000 & 0.000000000 \\ \mathrm{C} & 2.472478000 & -1.403793000 & 0.000000000 \\ \mathrm{C} & 1.217297000 & -0.717145000 & 0.000000000 \\ \mathrm{C} & 1.217297000 & 0.717145000 & 0.000000000 \\ \mathrm{C} & 2.472478000 & 1.403793000 & 0.000000000 \\ \mathrm{C} & 3.645689000 & 0.713759000 & 0.000000000 \\ \mathrm{C} & 0.000000000 & -1.399042000 & 0.000000000 \\ \mathrm{C} & 0.000000000 & 1.399042000 & 0.000000000 \\ \mathrm{C} & -1.217297000 & 0.717145000 & 0.000000000 \\ \mathrm{C} & -1.217297000 & -0.717145000 & 0.000000000 \\ \mathrm{C} & -2.472478000 & -1.403793000 & 0.000000000 \\ \mathrm{H} & -2.468023000 & -2.488511000 & 0.000000000 \\ \mathrm{C} & -3.645689000 & -0.713759000 & 0.000000000 \\ \mathrm{C} & -3.645689000 & 0.713759000 & 0.000000000 \\ \mathrm{C} & -2.472478000 & 1.403793000 & 0.000000000 \\ \mathrm{H} & 0.000000000 & -2.484946000 & 0.000000000 \\ \mathrm{H} & 4.590188000 & -1.244647000 & 0.000000000 \\ \mathrm{H} & 2.468023000 & -2.488511000 & 0.000000000 \\ \mathrm{H} & 2.468023000 & 2.488511000 & 0.000000000 \\ \mathrm{H} & 4.590188000 & 1.244647000 & 0.000000000 \\ \mathrm{H} & 0.000000000 & 2.484946000 & 0.000000000 \\ \mathrm{H} & -4.590188000 & -1.244647000 & 0.000000000 \\ \mathrm{H} & -4.590188000 & 1.244647000 & 0.000000000 \\ \mathrm{H} & -2.468023000 & 2.488511000 & 0.000000000\end{array}$

\section{benz-a-anthracene}

$\begin{array}{lrrr}\mathrm{C} & 4.740104000 & -0.007791000 & 0.000001000 \\ \mathrm{C} & 3.798332000 & 0.979600000 & 0.000000000 \\ \mathrm{C} & 2.409119000 & 0.659632000 & 0.000000000 \\ \mathrm{C} & 2.015350000 & -0.711033000 & 0.000000000 \\ \mathrm{C} & 3.026994000 & -1.715795000 & 0.000001000 \\ \mathrm{C} & 4.348613000 & -1.375548000 & 0.000001000 \\ \mathrm{C} & 1.410785000 & 1.643482000 & 0.000000000 \\ \mathrm{C} & 0.648453000 & -1.034516000 & 0.000000000 \\ \mathrm{C} & -0.339104000 & -0.061447000 & 0.000000000 \\ \mathrm{C} & 0.063604000 & 1.315107000 & 0.000000000 \\ \mathrm{C} & -0.942020000 & 2.350556000 & -0.000001000 \\ \mathrm{H} & -0.609757000 & 3.382983000 & -0.000001000 \\ \mathrm{C} & -2.254449000 & 2.048831000 & -0.000001000 \\ \mathrm{C} & -2.708932000 & 0.677953000 & -0.000001000 \\ \mathrm{C} & -1.770077000 & -0.377963000 & 0.000000000 \\ \mathrm{H} & 1.699846000 & 2.690158000 & 0.000000000 \\ \mathrm{H} & 5.793803000 & 0.244406000 & 0.000001000 \\ \mathrm{H} & 4.090993000 & 2.024070000 & 0.000000000 \\ \mathrm{H} & 2.724844000 & -2.757671000 & 0.000001000 \\ \mathrm{H} & 5.109241000 & -2.147120000 & 0.000001000 \\ \mathrm{H} & 0.386586000 & -2.085492000 & 0.000001000\end{array}$




$\begin{array}{lrrr}\mathrm{H} & -3.002926000 & 2.833818000 & -0.000001000 \\ \mathrm{C} & -2.257182000 & -1.697877000 & 0.000000000 \\ \mathrm{C} & -4.085892000 & 0.389408000 & -0.000001000 \\ \mathrm{C} & -3.611772000 & -1.962992000 & 0.000000000 \\ \mathrm{C} & -4.538027000 & -0.912264000 & 0.000000000 \\ \mathrm{H} & -4.789950000 & 1.214682000 & -0.000001000 \\ \mathrm{H} & -5.600688000 & -1.122690000 & 0.000000000 \\ \mathrm{H} & -3.957911000 & -2.989659000 & 0.000000000 \\ \mathrm{H} & -1.567461000 & -2.531536000 & 0.000000000\end{array}$

\section{benzene}

$\begin{array}{lrrr}\text { C } & 1.308082000 & -0.475132000 & 0.000000000 \\ \text { C } & 0.242564000 & -1.370375000 & 0.000000000 \\ \text { C } & -1.065518000 & -0.895247000 & 0.000000000 \\ \text { C } & -1.308081000 & 0.475133000 & 0.000000000 \\ \text { C } & -0.242565000 & 1.370375000 & 0.000000000 \\ \mathrm{C} & 1.065519000 & 0.895246000 & 0.000000000 \\ \mathrm{H} & 2.326651000 & -0.845105000 & 0.000001000 \\ \mathrm{H} & 0.431403000 & -2.437478000 & 0.000001000 \\ \mathrm{H} & -1.895228000 & -1.592360000 & 0.000000000 \\ \mathrm{H} & -2.326652000 & 0.845104000 & -0.000001000 \\ \mathrm{H} & -0.431401000 & 2.437478000 & 0.000000000 \\ \mathrm{H} & 1.895227000 & 1.592362000 & 0.000000000\end{array}$

\section{biphenyl}

$\begin{array}{lrrr}\mathrm{C} & -0.048881000 & 0.740342000 & 0.000197000 \\ \mathrm{C} & -0.517244000 & 1.424939000 & 1.125477000 \\ \mathrm{C} & -0.609032000 & 2.811751000 & 1.125642000 \\ \mathrm{C} & -0.233800000 & 3.537945000 & -0.000191000 \\ \mathrm{C} & 0.233800000 & 2.867170000 & -1.125816000 \\ \mathrm{C} & 0.325369000 & 1.480351000 & -1.125259000 \\ \mathrm{C} & 0.048881000 & -0.740342000 & 0.000197000 \\ \mathrm{C} & 0.517244000 & -1.424939000 & 1.125477000 \\ \mathrm{C} & 0.609032000 & -2.811751000 & 1.125642000 \\ \mathrm{C} & 0.233800000 & -3.537945000 & -0.000191000 \\ \mathrm{C} & -0.233800000 & -2.867170000 & -1.125816000 \\ \mathrm{C} & -0.325369000 & -1.480351000 & -1.125259000 \\ \mathrm{H} & -0.833995000 & 0.862219000 & 1.996582000 \\ \mathrm{H} & -0.982495000 & 3.325728000 & 2.003562000 \\ \mathrm{H} & -0.305281000 & 4.618996000 & -0.000328000 \\ \mathrm{H} & 0.536387000 & 3.425581000 & -2.003886000 \\ \mathrm{H} & 0.713456000 & 0.963993000 & -1.996224000 \\ \mathrm{H} & 0.833995000 & -0.862219000 & 1.996582000 \\ \mathrm{H} & 0.982495000 & -3.325728000 & 2.003562000 \\ \mathrm{H} & 0.305281000 & -4.618996000 & -0.000328000 \\ \mathrm{H} & -0.536387000 & -3.425581000 & -2.003886000 \\ \mathrm{H} & -0.713456000 & -0.963993000 & -1.996224000\end{array}$




\begin{tabular}{lrrr}
\multicolumn{4}{l}{ chrysene } \\
C & -4.654683000 & -0.074829000 & 0.000005000 \\
$\mathrm{C}$ & -3.790918000 & -1.140797000 & -0.000009000 \\
$\mathrm{C}$ & -2.391927000 & -0.940427000 & -0.000010000 \\
$\mathrm{C}$ & -1.863948000 & 0.373442000 & 0.000000000 \\
$\mathrm{C}$ & -2.781297000 & 1.451603000 & 0.000016000 \\
$\mathrm{C}$ & -4.138718000 & 1.234669000 & 0.000018000 \\
$\mathrm{C}$ & -1.496049000 & -2.051166000 & -0.000021000 \\
$\mathrm{C}$ & -0.425574000 & 0.558942000 & -0.000004000 \\
$\mathrm{C}$ & 0.425574000 & -0.558943000 & -0.000005000 \\
$\mathrm{C}$ & -0.152330000 & -1.868519000 & -0.000016000 \\
$\mathrm{C}$ & 1.863949000 & -0.373442000 & 0.000001000 \\
$\mathrm{C}$ & 2.391927000 & 0.940427000 & -0.000004000 \\
$\mathrm{C}$ & 1.496048000 & 2.051166000 & -0.000012000 \\
$\mathrm{C}$ & 0.152329000 & 1.868518000 & -0.000010000 \\
$\mathrm{H}$ & -1.912831000 & -3.052565000 & -0.000033000 \\
$\mathrm{H}$ & -5.725937000 & -0.235930000 & 0.000006000 \\
$\mathrm{H}$ & -4.168864000 & -2.157588000 & -0.000018000 \\
$\mathrm{H}$ & -2.424797000 & 2.472247000 & 0.000030000 \\
$\mathrm{H}$ & -4.816554000 & 2.079981000 & 0.000032000 \\
$\mathrm{H}$ & 0.488658000 & -2.738734000 & -0.000026000 \\
$\mathrm{H}$ & 1.912830000 & 3.052564000 & -0.000020000 \\
$\mathrm{H}$ & -0.488659000 & 2.738732000 & -0.000018000 \\
$\mathrm{C}$ & 2.781298000 & -1.451603000 & 0.000015000 \\
$\mathrm{C}$ & 3.790918000 & 1.140798000 & 0.000000000 \\
$\mathrm{C}$ & 4.138719000 & -1.234668000 & 0.000020000 \\
$\mathrm{C}$ & 4.654683000 & 0.074830000 & 0.000011000 \\
$\mathrm{H}$ & 2.424799000 & -2.472247000 & 0.000026000 \\
$\mathrm{H}$ & 4.816556000 & -2.079979000 & 0.000032000 \\
$\mathrm{H}$ & 5.725937000 & 0.235932000 & 0.000015000 \\
$\mathrm{H}$ & 4.168862000 & 2.157589000 & -0.000005000
\end{tabular}

\section{fluoranthene}

$\begin{array}{lrrr}\mathrm{C} & 0.000000000 & 2.420982000 & 2.158685000 \\ \mathrm{C} & 0.000000000 & 1.276781000 & 2.924860000 \\ \mathrm{C} & 0.000000000 & 0.000000000 & 2.297753000 \\ \mathrm{C} & 0.000000000 & 0.000000000 & 0.903804000 \\ \mathrm{C} & 0.000000000 & 1.169107000 & 0.107392000 \\ \mathrm{C} & 0.000000000 & 2.388292000 & 0.736051000 \\ \mathrm{H} & 0.000000000 & -1.346349000 & 4.007148000 \\ \mathrm{H} & 0.000000000 & 3.385572000 & 2.652730000 \\ \mathrm{H} & 0.000000000 & 1.346349000 & 4.007148000 \\ \mathrm{C} & 0.000000000 & -1.276781000 & 2.924860000 \\ \mathrm{C} & 0.000000000 & -1.169107000 & 0.107392000 \\ \mathrm{H} & 0.000000000 & 3.319205000 & 0.180165000 \\ \mathrm{C} & 0.000000000 & -2.388292000 & 0.736051000 \\ \mathrm{C} & 0.000000000 & -2.420982000 & 2.158685000 \\ \mathrm{H} & 0.000000000 & -3.319205000 & 0.180165000 \\ \mathrm{H} & 0.000000000 & -3.385572000 & 2.652730000 \\ \mathrm{C} & 0.000000000 & 0.709064000 & -1.296580000\end{array}$




$\begin{array}{lrrr}\mathrm{C} & 0.000000000 & 1.410713000 & -2.491757000 \\ \mathrm{C} & 0.000000000 & -0.709064000 & -1.296580000 \\ \mathrm{C} & 0.000000000 & 0.696615000 & -3.690740000 \\ \mathrm{H} & 0.000000000 & 2.494929000 & -2.499084000 \\ \mathrm{C} & 0.000000000 & -1.410713000 & -2.491757000 \\ \mathrm{C} & 0.000000000 & -0.696615000 & -3.690740000 \\ \mathrm{H} & 0.000000000 & 1.231285000 & -4.633096000 \\ \mathrm{H} & 0.000000000 & -2.494929000 & -2.499084000 \\ \mathrm{H} & 0.000000000 & -1.231285000 & -4.633096000\end{array}$

\section{fluorene}

$\begin{array}{lrrr}\mathrm{C} & -1.180201000 & -0.882959000 & 0.000000000 \\ \mathrm{C} & -0.735016000 & 0.447368000 & 0.000000000 \\ \mathrm{C} & -2.536706000 & -1.169334000 & 0.000001000 \\ \mathrm{C} & 0.000002000 & -1.827386000 & -0.000001000 \\ \mathrm{C} & -1.644388000 & 1.500024000 & -0.000001000 \\ \mathrm{C} & 0.735015000 & 0.447368000 & -0.000001000 \\ \mathrm{C} & -3.005029000 & 1.208058000 & 0.000000000 \\ \mathrm{H} & -1.303114000 & 2.529189000 & -0.000002000 \\ \mathrm{C} & -3.448437000 & -0.114448000 & 0.000001000 \\ \mathrm{H} & -3.728222000 & 2.014977000 & -0.000001000 \\ \mathrm{H} & -4.511744000 & -0.322922000 & 0.000001000 \\ \mathrm{H} & -2.887200000 & -2.195810000 & 0.000002000 \\ \mathrm{C} & 1.180202000 & -0.882959000 & -0.000001000 \\ \mathrm{C} & 2.536708000 & -1.169333000 & 0.000000000 \\ \mathrm{C} & 1.644385000 & 1.500024000 & 0.000000000 \\ \mathrm{C} & 3.005027000 & 1.208059000 & 0.000001000 \\ \mathrm{H} & 1.303111000 & 2.529189000 & 0.000000000 \\ \mathrm{C} & 3.448437000 & -0.114446000 & 0.000001000 \\ \mathrm{H} & 3.728218000 & 2.014979000 & 0.000001000 \\ \mathrm{H} & 4.511744000 & -0.322919000 & 0.000001000 \\ \mathrm{H} & 2.887204000 & -2.195808000 & 0.000000000 \\ \mathrm{H} & 0.000003000 & -2.475542000 & -0.881807000 \\ \mathrm{H} & 0.000004000 & -2.475544000 & 0.881803000\end{array}$

\section{naphthalene}

$\begin{array}{lrrr}\mathrm{C} & -2.424074000 & 0.707928000 & 0.000000000 \\ \mathrm{C} & -1.241715000 & 1.398323000 & 0.000000000 \\ \mathrm{C} & 0.000000000 & 0.710575000 & 0.000000000 \\ \mathrm{C} & 0.000000000 & -0.710575000 & 0.000000000 \\ \mathrm{C} & -1.241715000 & -1.398323000 & 0.000000000 \\ \mathrm{C} & -2.424074000 & -0.707928000 & 0.000000000 \\ \mathrm{H} & 1.236644000 & 2.483137000 & 0.000000000 \\ \mathrm{H} & -3.366419000 & 1.242696000 & 0.000000000 \\ \mathrm{H} & -1.236644000 & 2.483137000 & 0.000000000 \\ \mathrm{C} & 1.241715000 & 1.398323000 & 0.000000000 \\ \mathrm{C} & 1.241715000 & -1.398323000 & 0.000000000 \\ \mathrm{H} & -1.236644000 & -2.483137000 & 0.000000000\end{array}$




$\begin{array}{lrrr}\mathrm{H} & -3.366419000 & -1.242696000 & 0.000000000 \\ \mathrm{C} & 2.424074000 & -0.707928000 & 0.000000000 \\ \mathrm{C} & 2.424074000 & 0.707928000 & 0.000000000 \\ \mathrm{H} & 1.236644000 & -2.483137000 & 0.000000000 \\ \mathrm{H} & 3.366419000 & -1.242696000 & 0.000000000 \\ \mathrm{H} & 3.366419000 & 1.242696000 & 0.000000000\end{array}$

\section{phenanthrene}

$\begin{array}{lrrr}\mathrm{C} & 0.000000000 & 3.547794000 & -0.295109000 \\ \mathrm{C} & 0.000001000 & 2.827038000 & 0.875538000 \\ \mathrm{C} & 0.000001000 & 1.416443000 & 0.859452000 \\ \mathrm{C} & 0.000000000 & 0.728236000 & -0.377107000 \\ \mathrm{C} & 0.000000000 & 1.493531000 & -1.562652000 \\ \mathrm{C} & -0.000001000 & 2.870103000 & -1.525298000 \\ \mathrm{C} & 0.000001000 & 0.675609000 & 2.090538000 \\ \mathrm{C} & 0.000000000 & -0.728236000 & -0.377107000 \\ \mathrm{C} & -0.000001000 & -1.416443000 & 0.859452000 \\ \mathrm{C} & -0.000001000 & -0.675609000 & 2.090538000 \\ \mathrm{C} & -0.000001000 & -2.827038000 & 0.875538000 \\ \mathrm{H} & -0.000001000 & -3.335097000 & 1.833997000 \\ \mathrm{C} & 0.000000000 & -3.547794000 & -0.295109000 \\ \mathrm{C} & 0.000001000 & -2.870103000 & -1.525298000 \\ \mathrm{C} & 0.000000000 & -1.493531000 & -1.562652000 \\ \mathrm{H} & 0.000001000 & 1.229746000 & 3.022936000 \\ \mathrm{H} & 0.000000000 & 4.630875000 & -0.271611000 \\ \mathrm{H} & 0.000001000 & 3.335097000 & 1.833997000 \\ \mathrm{H} & -0.000001000 & 1.001646000 & -2.526192000 \\ \mathrm{H} & -0.000001000 & 3.432661000 & -2.451303000 \\ \mathrm{H} & -0.000001000 & -1.229746000 & 3.022936000 \\ \mathrm{H} & 0.000000000 & -4.630875000 & -0.271611000 \\ \mathrm{H} & 0.000001000 & -3.432661000 & -2.451303000 \\ \mathrm{H} & 0.000001000 & -1.001646000 & -2.526192000\end{array}$

\section{pyrene}

$\begin{array}{lrrr}\text { C } & 3.510932000 & 0.000000000 & 0.000362000 \\ \text { C } & 2.821541000 & -1.206647000 & 0.000302000 \\ \text { C } & 1.423632000 & -1.228767000 & 0.000159000 \\ \text { C } & 0.713365000 & 0.000000000 & 0.000074000 \\ \text { C } & 1.423632000 & 1.228767000 & 0.000136000 \\ \text { C } & 2.821541000 & 1.206647000 & 0.000280000 \\ \text { C } & 0.676265000 & -2.459888000 & 0.000093000 \\ \text { C } & -0.713365000 & 0.000000000 & -0.000074000 \\ \text { C } & -1.423632000 & -1.228767000 & -0.000136000 \\ \text { C } & -0.676265000 & -2.459888000 & -0.000047000 \\ \text { C } & -2.821541000 & -1.206647000 & -0.000280000 \\ 1 & -3.364798000 & -2.145363000 & -0.000327000 \\ \text { C } & -3.510932000 & 0.000000000 & -0.000362000 \\ \text { C } & -2.821541000 & 1.206647000 & -0.000302000 \\ \text { C } & -1.423632000 & 1.228767000 & -0.000159000\end{array}$




$\begin{array}{lrrc}\mathrm{C} & -0.676265000 & 2.459888000 & -0.000093000 \\ \mathrm{C} & 0.676265000 & 2.459888000 & 0.000047000 \\ \mathrm{H} & 1.227538000 & 3.394056000 & 0.000096000 \\ \mathrm{H} & -1.227538000 & 3.394056000 & -0.000160000 \\ \mathrm{H} & 1.227538000 & -3.394056000 & 0.000160000 \\ \mathrm{H} & 4.594449000 & 0.000000000 & 0.000473000 \\ \mathrm{H} & 3.364798000 & -2.145363000 & 0.000368000 \\ \mathrm{H} & 3.364798000 & 2.145363000 & 0.000327000 \\ \mathrm{H} & -1.227538000 & -3.394056000 & -0.000096000 \\ \mathrm{H} & -4.594449000 & 0.000000000 & -0.000473000 \\ \mathrm{H} & -3.364798000 & 2.145363000 & -0.000368000\end{array}$

\section{naphthacene}

$\begin{array}{lrrr}\mathrm{C} & -2.437829000 & -0.721350000 & 0.000021000 \\ \mathrm{C} & -1.231897000 & -1.402211000 & 0.000025000 \\ \mathrm{C} & 0.000000000 & -0.720503000 & 0.000011000 \\ \mathrm{C} & 0.000000000 & 0.720503000 & -0.000010000 \\ \mathrm{C} & -1.231897000 & 1.402211000 & -0.000016000 \\ \mathrm{C} & -2.437829000 & 0.721350000 & -0.000001000 \\ \mathrm{C} & 1.231897000 & -1.402211000 & 0.000015000 \\ \mathrm{C} & 1.231897000 & 1.402211000 & -0.000025000 \\ \mathrm{C} & 2.437829000 & 0.721350000 & -0.000020000 \\ \mathrm{C} & 2.437829000 & -0.721350000 & 0.000001000 \\ \mathrm{C} & 3.699854000 & -1.406789000 & 0.000006000 \\ \mathrm{H} & 3.695911000 & -2.491482000 & 0.000022000 \\ \mathrm{C} & 4.868715000 & -0.716802000 & -0.000009000 \\ \mathrm{C} & 4.868715000 & 0.716802000 & -0.000030000 \\ \mathrm{C} & 3.699854000 & 1.406789000 & -0.000036000 \\ \mathrm{H} & 1.232083000 & -2.488008000 & 0.000031000 \\ \mathrm{H} & -1.232083000 & -2.488008000 & 0.000041000 \\ \mathrm{H} & -1.232083000 & 2.488008000 & -0.000031000 \\ \mathrm{H} & 1.232083000 & 2.488008000 & -0.000041000 \\ \mathrm{H} & 5.814296000 & -1.245728000 & -0.000006000 \\ \mathrm{H} & 5.814296000 & 1.245728000 & -0.000042000 \\ \mathrm{H} & 3.695911000 & 2.491482000 & -0.000052000 \\ \mathrm{C} & -3.699854000 & -1.406789000 & 0.000036000 \\ \mathrm{C} & -3.699853000 & 1.406789000 & -0.000005000 \\ \mathrm{C} & -4.868715000 & -0.716802000 & 0.000030000 \\ \mathrm{C} & -4.868715000 & 0.716802000 & 0.000009000 \\ \mathrm{H} & -3.695911000 & -2.491482000 & 0.000052000 \\ \mathrm{H} & -5.814296000 & -1.245728000 & 0.000042000 \\ \mathrm{H} & -5.814296000 & 1.245728000 & 0.000005000 \\ \mathrm{H} & -3.695911000 & 2.491482000 & -0.000022000\end{array}$


ethenylbenzene

$\begin{array}{lrrr}\mathrm{C} & 0.510888000 & -0.222586000 & -0.057767000 \\ \mathrm{C} & 0.012631000 & 1.085319000 & -0.081647000 \\ \mathrm{C} & -1.353017000 & 1.324297000 & -0.026197000 \\ \mathrm{C} & -2.251068000 & 0.261489000 & 0.047628000 \\ \mathrm{C} & -1.770550000 & -1.042709000 & 0.059013000 \\ \mathrm{C} & -0.401698000 & -1.279673000 & 0.001965000 \\ \mathrm{C} & 1.953875000 & -0.523374000 & -0.096732000 \\ \mathrm{C} & 2.946081000 & 0.335389000 & 0.129770000 \\ \mathrm{H} & 0.697640000 & 1.921312000 & -0.160533000 \\ \mathrm{H} & -1.721380000 & 2.343233000 & -0.049217000 \\ \mathrm{H} & -3.316989000 & 0.451022000 & 0.088090000 \\ \mathrm{H} & -2.460690000 & -1.876557000 & 0.109792000 \\ \mathrm{H} & -0.029400000 & -2.298756000 & 0.010717000 \\ \mathrm{H} & 2.201597000 & -1.558952000 & -0.316946000 \\ \mathrm{H} & 3.979189000 & 0.015608000 & 0.078597000 \\ \mathrm{H} & 2.767178000 & 1.374178000 & 0.383296000\end{array}$

\section{ethylbenzene}

$\begin{array}{lrrr}\mathrm{C} & -0.241486000 & 0.496668000 & 0.000000000 \\ \mathrm{C} & -0.241155000 & -0.217334000 & 1.199051000 \\ \mathrm{C} & -0.241155000 & -1.608255000 & 1.202138000 \\ \mathrm{C} & -0.241057000 & -2.308506000 & 0.000000000 \\ \mathrm{C} & -0.241155000 & -1.608255000 & -1.202138000 \\ \mathrm{C} & -0.241155000 & -0.217334000 & -1.199051000 \\ \mathrm{C} & -0.185444000 & 2.004257000 & 0.000000000 \\ \mathrm{C} & 1.260081000 & 2.515350000 & 0.000000000 \\ \mathrm{H} & -0.244652000 & 0.324696000 & 2.139802000 \\ \mathrm{H} & -0.245513000 & -2.145711000 & 2.143336000 \\ \mathrm{H} & -0.244194000 & -3.391957000 & 0.000000000 \\ \mathrm{H} & -0.245513000 & -2.145711000 & -2.143336000 \\ \mathrm{H} & -0.244652000 & 0.324696000 & -2.139802000 \\ \mathrm{H} & -0.710477000 & 2.388678000 & -0.878577000 \\ \mathrm{H} & -0.710477000 & 2.388678000 & 0.878577000 \\ \mathrm{H} & 1.794122000 & 2.155127000 & 0.882098000 \\ \mathrm{H} & 1.794122000 & 2.155127000 & -0.882098000 \\ \mathrm{H} & 1.292397000 & 3.606827000 & 0.000000000\end{array}$

\section{indene}

$\begin{array}{lrrr}\text { C } & -2.277985000 & -0.010077000 & 0.000000000 \\ \text { C } & -1.854906000 & -1.338621000 & 0.000000000 \\ \mathrm{C} & -0.498278000 & -1.654257000 & 0.000000000 \\ \mathrm{C} & -1.349907000 & 1.032235000 & 0.000000000 \\ \mathrm{C} & 0.000000000 & 0.722638000 & 0.000000000 \\ \mathrm{C} & 0.426707000 & -0.615261000 & 0.000000000 \\ \mathrm{C} & 1.894915000 & -0.625872000 & 0.000000000 \\ \mathrm{C} & 2.352184000 & 0.634517000 & 0.000000000 \\ \mathrm{C} & 1.210687000 & 1.620147000 & 0.000000000\end{array}$




$\begin{array}{rrrr}\mathrm{H} & -3.338023000 & 0.214414000 & 0.000000000 \\ \mathrm{H} & -2.591210000 & -2.133744000 & 0.000000000 \\ \mathrm{H} & -0.172400000 & -2.688299000 & 0.000000000 \\ \mathrm{H} & -1.685080000 & 2.063864000 & 0.000000000 \\ \mathrm{H} & 2.499209000 & -1.523692000 & 0.000000000 \\ \mathrm{H} & 3.392297000 & 0.931617000 & 0.000000000 \\ \mathrm{H} & 1.237350000 & 2.271571000 & 0.880183000 \\ \mathrm{H} & 1.237350000 & 2.271571000 & -0.880183000\end{array}$

isopropenylbenzene

$\begin{array}{lrrr}\mathrm{C} & 1.852275000 & 1.215907000 & -0.233716000 \\ \mathrm{C} & 0.462810000 & 1.155806000 & -0.230217000 \\ \mathrm{C} & -0.204293000 & -0.053981000 & -0.011819000 \\ \mathrm{C} & 0.565409000 & -1.196558000 & 0.236734000 \\ \mathrm{C} & 1.952686000 & -1.137625000 & 0.237129000 \\ \mathrm{C} & 2.603343000 & 0.069291000 & -0.002800000 \\ \mathrm{H} & 2.347804000 & 2.162354000 & -0.415758000 \\ \mathrm{H} & -0.105152000 & 2.060002000 & -0.413651000 \\ \mathrm{H} & 0.067656000 & -2.133797000 & 0.456330000 \\ \mathrm{H} & 2.528172000 & -2.033738000 & 0.437955000 \\ \mathrm{H} & 3.685738000 & 0.116437000 & 0.000676000 \\ \mathrm{C} & -1.690092000 & -0.121459000 & -0.032253000 \\ \mathrm{C} & -2.330817000 & -1.216114000 & -0.447682000 \\ \mathrm{H} & -1.799093000 & -2.082278000 & -0.823094000 \\ \mathrm{H} & -3.413582000 & -1.264183000 & -0.442633000 \\ \mathrm{C} & -2.444755000 & 1.102826000 & 0.418853000 \\ \mathrm{H} & -2.076565000 & 1.454264000 & 1.385911000 \\ \mathrm{H} & -2.324285000 & 1.923783000 & -0.293345000 \\ \mathrm{H} & -3.510091000 & 0.888595000 & 0.502240000\end{array}$

\section{toluene}

$\begin{array}{rrrr}\mathrm{C} & 0.004183000 & 0.909663000 & 0.000000000 \\ \mathrm{C} & 0.007413000 & 0.194670000 & 1.198431000 \\ \mathrm{C} & 0.007413000 & -1.196238000 & 1.201423000 \\ \mathrm{C} & 0.006540000 & -1.897299000 & 0.000000000 \\ \mathrm{C} & 0.007413000 & -1.196238000 & -1.201423000 \\ \mathrm{C} & 0.007413000 & 0.194670000 & -1.198431000 \\ \mathrm{C} & -0.028475000 & 2.416411000 & 0.000000000 \\ \mathrm{H} & 0.012037000 & 0.734986000 & 2.139600000 \\ \mathrm{H} & 0.011913000 & -1.732736000 & 2.143147000 \\ \mathrm{H} & 0.009522000 & -2.980715000 & 0.000000000 \\ \mathrm{H} & 0.011913000 & -1.732736000 & -2.143147000 \\ \mathrm{H} & 0.012037000 & 0.734986000 & -2.139600000 \\ \mathrm{H} & -1.059986000 & 2.779654000 & 0.000000000 \\ \mathrm{H} & 0.465579000 & 2.821369000 & 0.884529000 \\ \mathrm{H} & 0.465579000 & 2.821369000 & -0.884529000\end{array}$




$\begin{array}{lrrr}\text { Coronene } & & & \\ \mathrm{C} & -3.66635400 & -0.75384600 & 0.00000000 \\ \mathrm{C} & -2.81825100 & 0.38811400 & 0.00000000 \\ \mathrm{C} & -1.41240200 & 0.19450700 & 0.00000000 \\ \mathrm{C} & -0.87464400 & -1.12591900 & 0.00000000 \\ \mathrm{C} & -1.74524800 & -2.24661700 & 0.00000000 \\ \mathrm{C} & -3.14985800 & -2.02207800 & 0.00000000 \\ \mathrm{C} & -0.53774800 & 1.32042400 & 0.00000000 \\ \mathrm{C} & 0.53774800 & -1.32042400 & 0.00000000 \\ \mathrm{C} & 1.41240200 & -0.19450700 & 0.00000000 \\ \mathrm{C} & 0.87464400 & 1.12591900 & 0.00000000 \\ \mathrm{C} & 2.81825100 & -0.38811400 & 0.00000000 \\ \mathrm{C} & 3.32610500 & -1.71681100 & 0.00000000 \\ \mathrm{C} & 2.48602800 & -2.79822300 & 0.00000000 \\ \mathrm{C} & 1.07301300 & -2.63473200 & 0.00000000 \\ \mathrm{C} & 0.17624300 & -3.73889100 & 0.00000000 \\ \mathrm{C} & -1.18031900 & -3.55207400 & 0.00000000 \\ \mathrm{H} & -1.84785300 & -4.40741200 & 0.00000000 \\ \mathrm{H} & 0.58772400 & -4.74282700 & 0.00000000 \\ \mathrm{H} & -4.74086400 & -0.60340200 & 0.00000000 \\ \mathrm{H} & -3.81354000 & -2.88040900 & 0.00000000 \\ \mathrm{H} & 4.40128200 & -1.86241100 & 0.00000000 \\ \mathrm{H} & 2.89299700 & -3.80399800 & 0.00000000 \\ \mathrm{C} & -3.32610500 & 1.71681100 & 0.00000000 \\ \mathrm{C} & -2.48602800 & 2.79822300 & 0.00000000 \\ \mathrm{C} & -1.07301300 & 2.63473200 & 0.00000000 \\ \mathrm{H} & -4.40128200 & 1.86241100 & 0.00000000 \\ \mathrm{H} & -2.89299700 & 3.80399800 & 0.00000000 \\ \mathrm{C} & -0.17624300 & 3.73889100 & 0.00000000 \\ \mathrm{C} & 1.18031900 & 3.55207400 & 0.00000000 \\ \mathrm{C} & 1.74524800 & 2.24661700 & 0.00000000 \\ \mathrm{H} & -0.58772400 & 4.74282700 & 0.00000000 \\ \mathrm{H} & 1.84785300 & 4.40741200 & 0.00000000 \\ \mathrm{C} & 3.66635400 & 0.75384600 & 0.00000000 \\ \mathrm{H} & 4.74086400 & 0.60340200 & 0.00000000 \\ \mathrm{C} & 3.14985800 & 2.02207800 & 0.00000000 \\ \mathrm{H} & 3.81354000 & 2.88040900 & 0.00000000\end{array}$

\section{triphenylene}

$\begin{array}{lrrr}\mathrm{C} & -1.75976900 & 3.32637500 & 0.00000700 \\ \mathrm{C} & -2.05756200 & 1.97792000 & -0.00006900 \\ \mathrm{C} & -1.04815700 & 0.99059300 & -0.00006900 \\ \mathrm{C} & 0.30712700 & 1.40914800 & 0.00001200 \\ \mathrm{C} & 0.58378200 & 2.79373700 & 0.00008700 \\ \mathrm{C} & -0.42263700 & 3.73933500 & 0.00008500 \\ \mathrm{C} & -1.37391500 & -0.43863700 & -0.00015700 \\ \mathrm{C} & 1.38216800 & 0.41244900 & 0.00001800 \\ \mathrm{C} & 1.06695100 & -0.97059200 & -0.00005800 \\ \mathrm{C} & -0.33375200 & -1.40313600 & -0.00014900 \\ \mathrm{C} & 2.12774600 & -1.90248700 & -0.00004700 \\ \mathrm{H} & 1.91513100 & -2.96242800 & -0.00010600\end{array}$




$\begin{array}{lrrr}\mathrm{C} & 3.44987400 & -1.50375500 & 0.00003600 \\ \mathrm{C} & 3.76083000 & -0.13930000 & 0.00011100 \\ \mathrm{C} & 2.74194500 & 0.79283900 & 0.00010100 \\ \mathrm{H} & -2.55993200 & 4.05776000 & 0.00000500 \\ \mathrm{H} & -3.09825300 & 1.68557100 & -0.00012800 \\ \mathrm{H} & 1.60801700 & 3.13950000 & 0.00014900 \\ \mathrm{H} & -0.17429100 & 4.79457100 & 0.00014500 \\ \mathrm{H} & 4.23947600 & -2.24653200 & 0.00004200 \\ \mathrm{H} & 4.79428500 & 0.18801300 & 0.00017700 \\ \mathrm{H} & 3.00924500 & 1.84032300 & 0.00016100 \\ \mathrm{C} & -0.68448100 & -2.77086900 & -0.00022300 \\ \mathrm{C} & -2.71139700 & -0.89114600 & -0.00025000 \\ \mathrm{C} & -2.00123700 & -3.18698300 & 0.00016600 \\ \mathrm{C} & -3.02742600 & -2.23547500 & 0.00020200 \\ \mathrm{H} & 0.08853900 & -3.52650200 & -0.00016600 \\ \mathrm{H} & -2.23448000 & -4.24565300 & 0.00036200 \\ \mathrm{H} & -4.06548300 & -2.54783700 & 0.00049200 \\ \mathrm{H} & -3.52280300 & -0.17689200 & 0.00004000\end{array}$

\section{biphenylene}

$\begin{array}{lrrr}\mathrm{C} & 0.75441200 & -0.71102400 & 0.00000000 \\ \mathrm{C} & 0.75441200 & 0.71102400 & 0.00000000 \\ \mathrm{C} & 1.91411700 & 1.44306800 & 0.00000000 \\ \mathrm{C} & 3.11901400 & 0.69410000 & 0.00000000 \\ \mathrm{C} & 3.11901400 & -0.69410000 & 0.00000000 \\ \mathrm{C} & 1.91411700 & -1.44306800 & 0.00000000 \\ \mathrm{C} & -0.75441200 & -0.71102400 & 0.00000000 \\ \mathrm{C} & -0.75441200 & 0.71102400 & 0.00000000 \\ \mathrm{C} & -1.91411700 & 1.44306800 & 0.00000000 \\ \mathrm{C} & -3.11901400 & 0.69410000 & 0.00000000 \\ \mathrm{C} & -3.11901400 & -0.69410000 & 0.00000000 \\ \mathrm{C} & -1.91411700 & -1.44306800 & 0.00000000 \\ \mathrm{H} & 1.93424400 & 2.52666700 & 0.00000000 \\ \mathrm{H} & 4.06673600 & 1.22094200 & 0.00000000 \\ \mathrm{H} & 4.06673600 & -1.22094200 & 0.00000000 \\ \mathrm{H} & 1.93424400 & -2.52666700 & 0.00000000 \\ \mathrm{H} & -1.93424400 & 2.52666700 & 0.00000000 \\ \mathrm{H} & -4.06673600 & 1.22094200 & 0.00000000 \\ \mathrm{H} & -4.06673600 & -1.22094200 & 0.00000000 \\ \mathrm{H} & -1.93424400 & -2.52666700 & 0.00000000\end{array}$

\section{phenylethyne}

$\begin{array}{lrrr}\text { C } & -2.02197900 & -0.00001000 & 0.00000600 \\ \mathrm{C} & -3.22702500 & 0.00000200 & -0.00001700 \\ \mathrm{C} & -0.59379400 & -0.00000400 & 0.00000400 \\ \mathrm{C} & 0.11925200 & 1.21089400 & 0.00000200 \\ \mathrm{C} & 1.50996400 & 1.20654600 & -0.00000100 \\ \mathrm{C} & 2.20963400 & 0.00000500 & -0.00000200 \\ \mathrm{C} & 1.50997300 & -1.20654000 & -0.00000100 \\ \mathrm{C} & 0.11926100 & -1.21089800 & 0.00000200 \\ \mathrm{H} & -4.28949800 & 0.00001900 & 0.00003600\end{array}$




$\begin{array}{rrrr}\mathrm{H} & -0.42694800 & 2.14639700 & 0.00000300 \\ \mathrm{H} & 2.04898700 & 2.14717600 & -0.00000200 \\ \mathrm{H} & 3.29367100 & 0.00000900 & -0.00000500 \\ \mathrm{H} & 2.04900300 & -2.14716600 & -0.00000200 \\ \mathrm{H} & -0.42693300 & -2.14640500 & 0.00000300\end{array}$

\section{Ovalene}

$\begin{array}{lrrr}\mathrm{C} & 2.46112600 & -0.71236400 & 0.00000100 \\ \mathrm{C} & 1.22629200 & -1.42445900 & 0.00000100 \\ \mathrm{C} & 0.00000000 & -0.71659000 & 0.00000000 \\ \mathrm{C} & 0.00000000 & 0.71659000 & 0.00000000 \\ \mathrm{C} & 1.22629200 & 1.42445900 & 0.00000000 \\ \mathrm{C} & 2.46112600 & 0.71236400 & 0.00000100 \\ \mathrm{C} & -1.22629200 & -1.42445900 & 0.00000000 \\ \mathrm{C} & -1.22629200 & 1.42445900 & -0.00000100 \\ \mathrm{C} & -2.46112600 & 0.71236400 & -0.00000100 \\ \mathrm{C} & -2.46112600 & -0.71236400 & -0.00000100 \\ \mathrm{C} & -3.69181500 & 1.42102800 & -0.00000100 \\ \mathrm{C} & -3.66013800 & 2.85243000 & -0.00000100 \\ \mathrm{C} & -2.48306600 & 3.53723900 & -0.00000100 \\ \mathrm{C} & -1.22260800 & 2.85433200 & -0.00000100 \\ \mathrm{C} & 0.00000000 & 3.53458800 & -0.00000100 \\ \mathrm{C} & 1.22260800 & 2.85433200 & 0.00000000 \\ \mathrm{H} & 0.00000000 & 4.62019000 & -0.00000100 \\ \mathrm{H} & -4.60321000 & 3.38901700 & -0.00000200 \\ \mathrm{H} & -2.48170000 & 4.62213700 & -0.00000100 \\ \mathrm{C} & 1.22260800 & -2.85433200 & 0.00000100 \\ \mathrm{C} & 0.00000000 & -3.53458800 & 0.00000100 \\ \mathrm{C} & -1.22260800 & -2.85433200 & 0.00000000 \\ \mathrm{H} & 0.00000000 & -4.62019000 & 0.00000100 \\ \mathrm{C} & -2.48306600 & -3.53723900 & 0.00000000 \\ \mathrm{C} & -3.66013800 & -2.85243000 & -0.00000100 \\ \mathrm{C} & -3.69181500 & -1.42102800 & -0.00000100 \\ \mathrm{H} & -2.48170000 & -4.62213700 & 0.00000000 \\ \mathrm{H} & -4.60321000 & -3.38901700 & -0.00000100 \\ \mathrm{C} & -4.90013000 & 0.68884100 & -0.00000100 \\ \mathrm{H} & -5.84001500 & 1.23084300 & -0.00000200 \\ \mathrm{C} & -4.90013000 & -0.68884100 & -0.00000100 \\ \mathrm{H} & -5.84001500 & -1.23084300 & -0.00000100 \\ \mathrm{H} & 2.48306600 & 3.53723900 & 0.00000000 \\ \mathrm{C} & 3.69181500 & 1.42102800 & 0.00000100 \\ \mathrm{C} & 3.69181500 & -1.42102800 & 0.00000100 \\ \mathrm{C} & 2.48306600 & -3.53723900 & 0.00000100 \\ \mathrm{C} & 3.66013800 & 2.85243000 & 0.00000100 \\ \mathrm{C} & 4.90013000 & 0.68884100 & 0.00000100 \\ \mathrm{C} & 4.90013000 & -0.68884100 & 0.00000100 \\ \mathrm{C} & 3.66013800 & -2.85243000 & 0.00000100 \\ \mathrm{H} & 2.48170000 & 4.62213700 & 0.00000000 \\ \mathrm{H} & 5.60321000 & 3.38901700 & 0.00000100 \\ \mathrm{H} & & 1.23084300 & 0.00000100 \\ \mathrm{H} & & -1.23084300 & 0.00000200\end{array}$


$\mathrm{H} \quad 4.60321000 \quad-3.38901700 \quad 0.00000200$

$\mathrm{H} \quad 2.48170000 \quad-4.62213700 \quad 0.00000100$ 